\title{
TEG Cascaded Solar PV System with Enhanced Efficiency by Using the PSO MPPT Boost Converter
}

\author{
Chinchinada V. S. L. Kalyani ${ }^{1 *}$, Motepalli Sunil Kumar ${ }^{2}$, Tella Nagaraju ${ }^{3}$ \\ ${ }^{I}$ M.Tech. Scholar, Department of Electrical and Electronics Engineering, Sir C. R. Reddy College of \\ Engineering, Eluru, India \\ ${ }^{2,3}$ Assistant Professor, Department of Electrical and Electronics Engineering, Sir C. R. Reddy College of \\ Engineering, Eluru, India \\ *Corresponding author: kalyani.chinchinada666@gmail.com
}

\begin{abstract}
Thermoelectric generators (TEGs) are used in small power applications to generate electrical energy from waste heats. Maximum power is obtained when the connected load to the ends of TEGs matches their internal resistance. However, impedance matching cannot always be ensured. Therefore, TEGs operate at lower efficiency. For this reason, maximum power point tracking (MPPT) algorithms are utilized. In this study, both TEGs and a boost converter with MPPT were modeled together. Detailed modeling, simulation, and verification of TEGs depending on the Seebeck coefficient, the hot/cold side temperatures, and the number of modules in MATLAB/Simulink were carried out. In addition, a boost converter having a particle swarm optimization (PSO) MPPT algorithm was added to the TEG modeling. After the TEG output equations were determined, the TEG modeling was performed based on manufacturer data sheets. Thanks to the TEG model and the boost converter with PSO MPPT, the maximum power was tracked with a value of $98.64 \%$ and the power derived from the TEG was nearly unaffected by the load changes. The power outputs obtained from the system with and without MPPT were compared to emphasize the importance of MPPT. These simulation values were verified by using an experimental setup. Ultimately, the proposed modeling provides a system of TEGs and a boost converter having PSO MPPT.
\end{abstract}

Keywords: Modeling, MATLAB/Simulink, PSO MPPT, Thermoelectric Generator.

\section{Introduction}

Thermoelectric generators (TEGs) are semiconductor devices that convert the temperature difference between surfaces into electrical energy directly. In order to increase their voltage, thermos elements (TEs) of $p$ and $n$ types are electrically connected in series and thermally connected in parallel to raise thermal conductivity. They are utilized as small power electric energy sources both to gain electrical energy from waste heats and to contribute to energy efficiency.

TEGs have some advantages and disadvantages. Their advantages are that there are no moving parts, they have a long service life, and they operate quietly. Moreover, they do not release greenhouse gases and therefore do not have any harmful effects on the environment. However, they have two disadvantages, which are low efficiency and high cost. For these reasons, the usage areas are limited. However, considering the contribution of TEGs to energy efficiency, the generated electricity is quite valuable.

When a load is directly connected to TEG terminals, if the load resistance and the internal resistance of the TEG are not equal, TEG efficiency decreases further. This is called an impedance imbalance. In order to avoid this, they are used with converters that perform both maximum power point tracking (MPPT) and power regulation as in photovoltaic panels (PVs). Various algorithms have been developed for the MPPT process in these converters. It is observed that these algorithms are adapted from PVs. The most common of these is the particle swarm optimization (PSO) algorithm. The algorithm is a reference for other algorithms and the results of the developed algorithms are compared with those of the algorithm. The focus of the present study is on detailed modeling of a TEG with the Seebeck coefficient and temperature difference provided by the TEG manufacturers, as well as the minimization of impedance imbalance by means of a boost converter with PSO MPPT.

TEG manufacturers publish datasheets of TEGs. The datasheets include maximum power, current, and voltage when the impedance matching is provided. Furthermore, they ensure hot and cold surface temperatures that can be reached by TEGs. In addition, they indicate the Seebeck coefficient, which is one of the most important criteria. When a TEG is modeled via temperature difference and Seebeck coefficient, the power value taken from the TEG can be calculated easily. Furthermore, the power of TEGs is low. For this reason, they must be connected in series and parallel to achieve the desired power value. They are connected in series in order to increase voltage and connected in parallel in order to raise current. If the numbers of series and parallel connected TEGs are arranged in modeling, ideal modeling will be carried out for industrial TEG users.

In the literature, TEG models have been given with the 
product characteristics of the manufacturers. In these studies, hot and cold side temperatures, power, voltage, and current matching and optimal efficiency values were used together for the TEG models. The responses of TEGs were measured by observing the changes in rapidly changing temperature conditions. Moreover, the efficiency values obtained from the series/parallel connection of TEGs with each other were determined. In addition, TEGs have been utilized in both thermoelectric coolers (TECs) and PVs to improve energy efficiency in grid-on and grid-off applications. They contribute to increase the energy efficiency of PV panels throughout by converting the temperature difference between the heated and the relatively cold surfaces of PV into electrical energy.

When the modeling studies are examined carefully, the detailed modeling's of PV by MATLAB/Simulink are quite common and these are the basis of other studies. However, it appears that TEGs have not been modeled in detail by means of Seebeck coefficient and cold-hot side temperature values. Therefore, the present study is based on the modeling of TEGs with these parameters so that MPPT applications can be implemented easily via SimPowerSystems in MATLAB /Simulink. Additionally, the present study provides boost converter modeling with a PSO MPPT algorithm. Finally, the results of TEG modeling with and without MPPT have been compared and confirmed by means of an experimental liquidliquid TEG system setup newly installed.

One of the most important features of the present paper, which differs from the studies in the literature, is to simulate TEGs by entering the Seebeck coefficient, the hot/cold side temperatures, and the number of modules, as well as the addition of MPPT to these simulation studies. There are some models in the literature related to TEGs. However, when these studies are taken into consideration, it is seen that there is no study involving comprehensive modeling. TEG modeling, boost converter modeling with and without PSO MPPT, and the verification of these simulation studies by means of an experimental setup will be a great source for researchers in this field.

The presentation of the present study is as follows. After giving the introduction section in the first part of the study, the TEG structure, equations, characteristic curves, a boost converter, and a PSO MPPT algorithm are expressed in the material section. The third section is divided into three parts. One of them is TEG modeling carried out by means of the hot/cold side temperatures and Seebeck coefficient. Second is series/parallel connected TEG modeling and the last one is TEG modeling with a boost converter having PSO MPPT. Then, in the fourth section, the modeling and the experimental outcomes and discussions are given. Finally, the results are explained at the end of the study.

\section{Material}

In this section, the structure, equations, and characteristic curves of TEGs, a boost converter, and a PSO MPPT algorithm are expressed.

\section{A. TEGs}

A TE is formed by combining high figure of merit $p$ and $n$ type semiconductors. The TEs are connected in series with each other to increase the voltage. TEGs are made up by connecting the TEs in parallel with the help of ceramic plates in order to raise thermal conductivity. When a temperature difference between the ceramic plates is generated, heat transfer from the hot surface to the cold surface becomes possible. The heat transfer leads to electron flow from the $n$-type to the $p$ type semiconductors. Thus, a voltage is generated as follows:

$$
V_{O C}=\alpha \cdot \Delta T,
$$

where $V_{O C}$ is the open circuit voltage $(\mathrm{V})$ of the TEG, $\alpha$ is the Seebeck coefficient $(\mathrm{V} / \mathrm{K})$, and $\Delta T$ is the temperature difference (K). The temperature difference is between the hot, $T_{h}$, and the cold surfaces, Tc, $\left(\Delta T=T_{h}-T_{c}\right)$. The Seebeck coefficient of a good material varies between 100 and $300 \mu \mathrm{V} / \mathrm{K}$. The temperature value is determined according to the catalog information provided by manufacturers. The TEGs used for industrial purposes have a hot surface temperature of up to 350 - C. The value is higher in TEGs used in vehicle exhaust systems.

The electrical equivalent circuit of a TEG consists of a temperature-dependent voltage source and an internal resistor, $R_{i n}$. A load, $R_{L}$, is connected to the TEG for generating power. When the load value and the internal resistance of the TEG match $\left(R_{i n}=R_{L}\right)$, the power generated from the TEG reaches a maximum power point (MPP). As the load value changes, the generated power decreases. When the load is infinite, an opencircuit voltage is measured from the TEG terminals. Similarly, the load is zero when TEG terminals are shorted. Therefore, a short-circuit current passes through the TEG. When the power taken from a TEG is at the MPP point, the open-circuit voltage and the short-circuit current are also in their half-value as described below:

$$
\begin{aligned}
& V_{M P P}=V_{O C} / 2 \\
& I_{M P P}=I_{S C} / 2
\end{aligned}
$$

where $V_{M P P}$ and $I_{M P P}$ are the voltage $(\mathrm{V})$ and the current (A) at the MPP, respectively. $I_{S C}$ is the short- circuit current (A). The MPP can be defined with these values. The value of the current passing through the TEG is given below:

$$
I_{T E G}=V_{O C} /\left(R_{i n t}+R_{L}\right)
$$

where $I_{T E G}, R_{\text {int }}$, and $R_{L}$ are the current of TEG (A), the internal resistance of TEG $(\Omega)$, and the load resistance $(\Omega)$, respectively.

Depending on the load resistance and the internal resistance, the power taken from the TEG is as follows: 


$$
P=V_{O C} 2 . R_{L} /\left(R_{\text {int }}+R_{L}\right) 2
$$

Where $P$ is the power generated from the TEG (W). The power is sensitive to load changes; at the same time, it is directly proportional to the temperature difference. As the temperature difference is raised, the power value also increases and, as a result, the MPP changes. If an equal and constant load to the internal resistance of the TEG is connected, the MPP value can be captured for a given temperature difference. However, as the temperature difference varies, the MPP shifts from the old MPP. The load must be adaptive so that the MPP value can be tracked continuously. For this purpose, an adaptation circuit is placed between the TEG and the load. The circuits are converters performing both power regulation and MPPT.

\section{B. Boost converter}

DC-DC converters are electronic devices that efficiently change the voltage level from one value to another. They are in the group of not insulated converters that increase the DC voltage level from low to high. A boost converter is illustrated in figure 1. The coil of the converter is very important for MPPs. The MPP points are captured by programmable coil current. The block diagram of a TEG system with MPPT is also presented in the given in figure 2 .

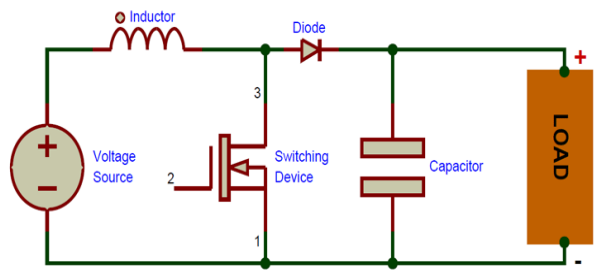

Fig. 1. Boost converter

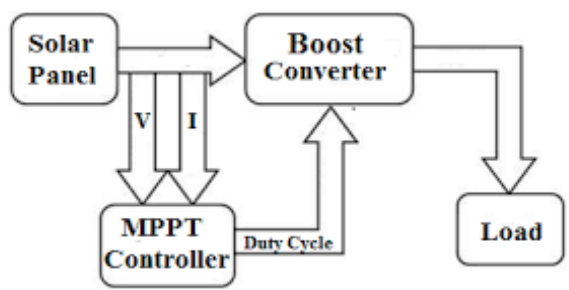

Fig. 2. Block diagram

The voltage in a boost converter is raised by switching the $\mathrm{S} 1$ switch from a few $\mathrm{kHz}$ to a few $\mathrm{MHz}$. The ideal switching frequency is between $20 \mathrm{kHz}$ and $2 \mathrm{MHz}$. The output voltage is regulated by the duty cycle of the switch. When the S1 switch is switched on, the current flows through the $\mathrm{L} 1$ coil and the $\mathrm{S} 1$ switch, and the energy is stored in the magnetic field of the coil. A current does not flow through the D1 diode and the load current is provided via the $\mathrm{C} 1$ capacitor. When the $\mathrm{S} 1$ switch is switched off, the drop in the L1 coil current is immediately supplied with its back-electromotive force and the L1 coil voltage is added to the source voltage. The current flows through the L1 coil, the $\mathrm{D} 1$ diode, and the $R_{L}$ load, and thereby the capacitor $\mathrm{C} 1$ is charged. Therefore, the output voltage is greater than the input voltage and the voltage equation is as follows:

$$
V_{\text {out }} / V_{\text {in }}=1 /(1-D) \text {, }
$$

Where $V_{\text {out }}, V_{\text {in }}$, and $D$ are the output voltage, the input voltage, and the duty cycle, respectively. The $1-D$ value is proportional to the on position time of the S1 switch. The boost rate is as follows:

$$
V_{\text {ou }} / V_{\text {in }}=T / T_{\text {off }}
$$

Where $T$ is the switching cycle (s) and $T_{\text {off }}$ is the off cycle of switching (s).

\section{Particle Swarm Optimization}

PSO is a swarm intelligence optimization algorithm developed by Eberhart and Kennedy in 1995, which is inspired by the social behavior of bird flocking and fish schooling. PSO is a global optimization algorithm for dealing with problems on which a point or surface in an $\mathrm{n}$-dimensional space represents a best solution. In this algorithm, several cooperative agents are used, and each agent exchanges information obtained in its respective search process. Each agent, referred to as a particle, follows two very simple rules, i.e., to follow the best performing particle, and to move toward the best conditions found by the particle itself. By this way, each particle ultimately evolves to an optimal or close to optimal solution.

The genetic algorithm (GA) is the most popular of the socalled evolutionary methods in the electromagnetics community. Recently, a new stochastic algorithm called particle swarm optimization (PSO) has been shown to be a valuable addition to the electromagnetic design engineer's toolbox.

The standard PSO method can be defined using the following equations:

$$
\begin{aligned}
& \mathrm{v}_{\mathrm{i}}(\mathrm{k}+1)=\mathrm{wv}_{\mathrm{i}}(\mathrm{k})+\mathrm{c} 1 \mathrm{r} 1 \cdot\left(\text { pbest }, \mathrm{i}-\mathrm{x}_{\mathrm{i}}(\mathrm{k})\right)+\mathrm{c} 2 \mathrm{r} 2 \cdot\left(\text { gbest }-\mathrm{x}_{\mathrm{i}}(\mathrm{k})\right) \\
& \mathrm{x}_{\mathrm{i}}(\mathrm{k}+1)=\mathrm{x}_{\mathrm{i}}(\mathrm{k})+\mathrm{v}_{\mathrm{i}}(\mathrm{k}+1) \\
& \mathrm{i}=1,2, \ldots, \mathrm{N}
\end{aligned}
$$

Where $x_{i}$ is the position of particle $i$; vi is the velocity of particle $\mathrm{i}$; $\mathrm{k}$ denotes the iteration number; $\mathrm{w}$ is the inertia weight; $\mathrm{r} 1$ and $\mathrm{r} 2$ are random variables uniformly distributed within [0,1]; and $\mathrm{c} 1, \mathrm{c} 2$ are the cognitive and social coefficient, respectively. The variable pbest,i is used to store the best position that the $\mathrm{i}^{\text {th }}$ particle has found so far, and gbest is used to store the best position of all the particles. PSO is used to perform on-time switching which handles the shading problem. The dispersing of shade can be done for a TCT-interconnected PV array using a PSO algorithm. The shading can be initialized randomly and row current is obtained for each row. Based on the row current, the velocity changes for each PV cells within 
the column arrangements. For each iteration, the row current is minimized. The difference in row current should be close to zero to locate global optimum solutions. When the solution is reached, the velocity change in each column is reduced based on velocity/number. Hence, the PSO method is efficient for performing on-time switching. The flowchart of a basic PSO algorithm is illustrated in fig. 3 .

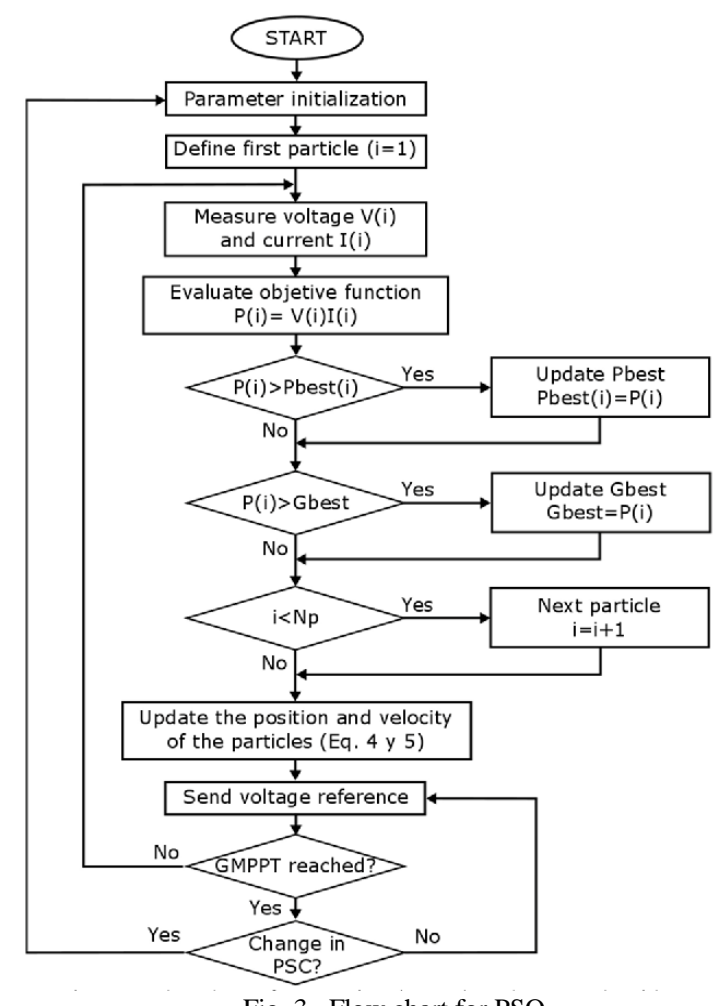

Fig. 3. Flow chart for PSO

It is well known that photovoltaic (PV) panel present a nonlinear relationship between its current and voltage quantities. Furthermore, when PV panels are under partial shading, the characteristic curve $\mathrm{I}_{\mathrm{pv}} \mathrm{XV}_{\mathrm{pv}}$ can present, simultaneously, several local and global maximum power points. Therefore, the proposed MPPT-PSO algorithm deals to overcome the partial shading effects, tracking the global maximum power point in order to maximize the power extraction of the PV arrangements.

This paper presents an improved control strategy to extract the maximum power available in the PV system by using the Particle Swarm Optimization (PSO) as MPPT based technique.

\section{Experimental Setup}

When a TEG is used alone in a system as a single module, the obtained power value is low. To increase this power value, they should be connected in series or parallel. A setup of 40 TEGs was used in the setup. Those were placed between three aluminum plates. Twenty of them were connected in series in order to increase the voltage value. Additionally, in order to enhance the current value, these two groups of 20 were connected in parallel. In the experimental installation, hot and cold water were passed from the middle and outside aluminum plates, respectively, for providing the temperature difference. Thus, electric energy was generated by the obtained temperature difference.

In order to compare the simulation with the experimental results. The input/output current/voltage values of the TEG system were measured by four of them. The current and voltage measurement error values of the measuring instruments were $1.2 \%$ and $0.6 \%$, respectively. With the help of another measuring device, both the hot and cold water outlet temperature values of the TEG system were taken. The measurement error value of the measuring instrument was $0.4 \%$. A K-type point thermocouple was used to measure the temperature values. As a hot water input source in the system, a Baymak brand thermosiphon was preferred. The device increased the water temperature to about $90^{\circ} \mathrm{C}$. The temperature value of the hot water was taken from the temperature indicator on the thermosiphon. The measurement error value of this was $0.5 \%$. For the cold water inlet of the system, running water was used. Four stone resistors were used to see the system output power values depending on load changes. During the experiments, the flow rates of the hot and cold water entering the TEG system were kept constant and they were taken out via the wash basin after use.

\section{Method}

In this section, the modeling of TEGs with Seebeck coefficient and temperature values given the datasheets of manufacturers, the TEG modeling in series and parallel without MPPT, and the TEG modeling in series and parallel with a PSO MPPT boost converter by means of MATLAB/Simulink are explained.

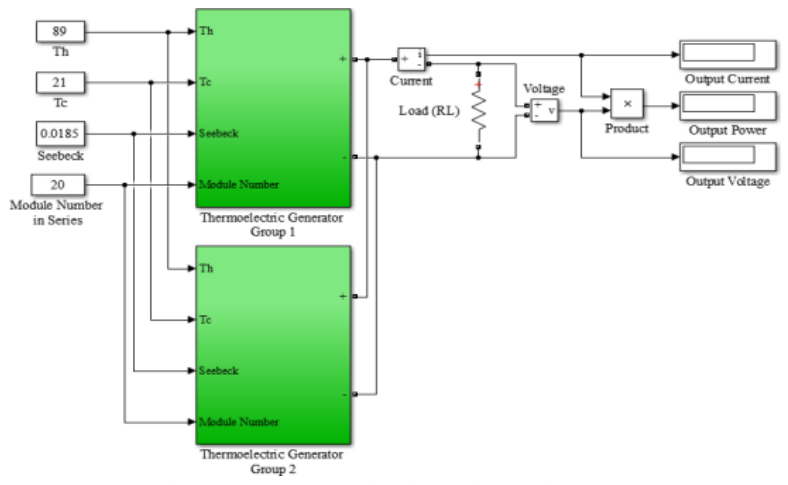

Fig. 4. TEG Modeling in series and parallel

\section{A. TEG modeling with MATLAB/Simulink}

In the present study, the TEGs manufactured by TES Thermoelectric Systems were utilized. Their properties are given below:

Hot surface temperature, $T_{h}=250{ }^{\circ} \mathrm{C}$

Cold surface temperature, $T_{c}=30{ }^{\circ} \mathrm{C}$

Open circuit voltage, $V_{O C}=4 \pm 0.4$ 
Short circuit current, $I_{S C}=5.8 \pm 0.6$

Load matching resistance, $R_{\text {in }}=R_{L} 0.7 \Omega$

Load matching output voltage $=2.0 \pm 0.2$

Load matching output current $=2.9 \pm 0.3$

Load matching output power $R_{\text {in }}=R_{L}=5.8 \mathrm{~W}$

Seebeck coefficient $=185 \mu \mathrm{V} / \mathrm{K}$

Figure of merit $=2.86 \times 10^{-3} \mathrm{~K}^{-1}$

$\mathrm{ZT}=0.8$

Modeling of a TEG modeled and simulated through MATLAB/Simulink is shown as a temperature-dependent voltage source in figure 5. In the modeling, the TEG output power was obtained by Seebeck coefficient and hot/cold side temperatures. In figure 5(a), one TEG was used. The temperature difference was calculated in the modeling. Moreover, the internal resistance value was entered in the modeling as shown figure 5(b). Here the TEG modeling is a temperature-dependent controlled voltage source.

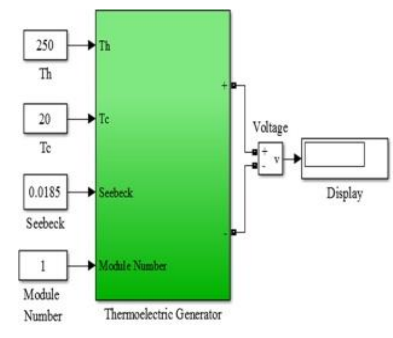

(a)

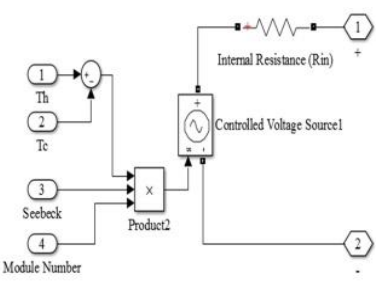

(b)
Fig. 5. For one TEG system, (a) modeling and (b) internal structure

B. TEG modeling in series and parallel without MPPT Boost Converter

Increasing the power generated from TEGs is carried out by series and parallel connection of the TEGs. The modeling of TEGs connected in series and parallel is depicted in figure 4. The number of connected modules in the TEG modeling was 20 in series. In this case, the internal resistance of this model was $0.7 \times 20=14 \Omega$. In order to increase the generated power, a separate model consisting of 20 TEGs was added to the series connected model in parallel. Thus, the TEG internal resistance value of the system was $14 / 2=7 \Omega$. In addition, current, voltage, and power indicators were put to measure the power to be generated depending on load and temperature variations.

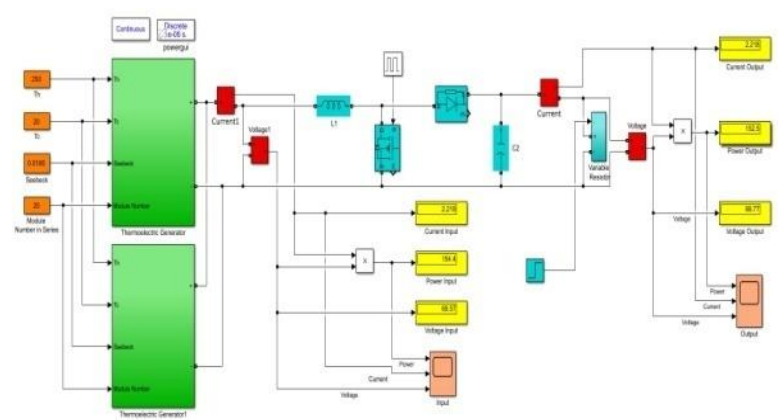

Fig. 6. TEG system modeling without a PSO MPPT boost converter
C. TEG modeling in series and parallel with a $P \& O M P P T$ boost converter

A boost converter with PSO MPPT was included in the designed modeling as shown in figure 7. Its parameter values were selected as $\mathrm{L} 1=1 \mathrm{mH}, \mathrm{C} 1=3000 \mu \mathrm{F}$, and switching frequency $\mathrm{f}=20 \mathrm{kHz}$. The power variations generated from the TEG system in series and parallel were minimized by the modeling.

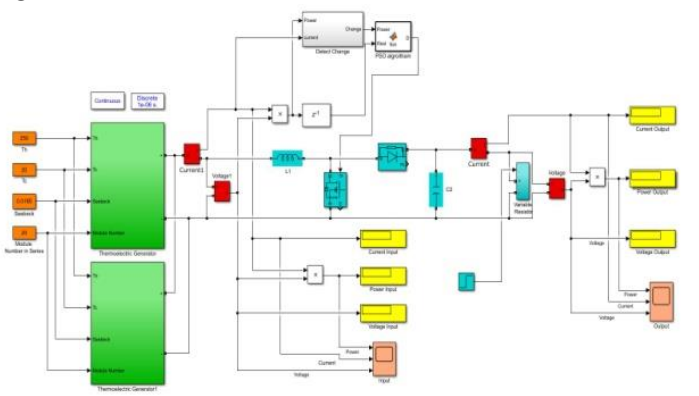

Fig. 7. TEG system modeling with a PSO MPPT boost converter

In figure 7, a variable resistor was connected to the boost converter output. That was changed to determine the MPPT of the boost converter at different load variations. In addition, a variable temperature signal was applied to the hot side input in order to determine the reaction of the boost converter with PSO MPPT at different temperatures.

In figure 8, for determining the value of MPPT, the current, voltage, and power indicators were connected to both the TEG output and the output of the boost converter with PSO MPPT. Lastly, two oscilloscopes were put to measure the time responses of both the TEG output and the boost converter with PSO MPPT.

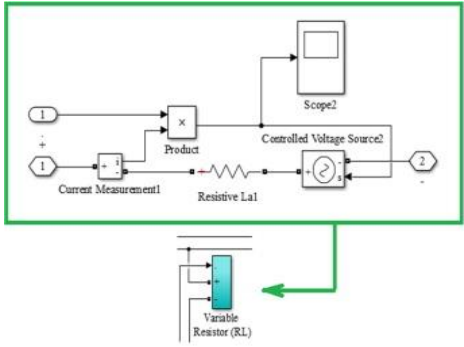

(a)

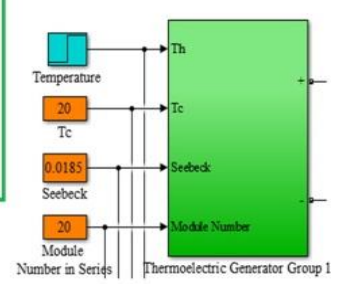

(b)
Fig. 8. Structure of (a) Variable resistor, (b) variable hot side temperature

\section{Results}

Firstly, as in the MATLAB/Simulink design shown in figure 4 , the load resistance was directly connected to the TEG terminals and the results were obtained from the experimental setup. Then, as shown in figure 6,7 , the results of the boost converter with PSO MPPT were taken from the experimental setup. The internal resistance of the TEG system was $7 \Omega$. In the experiments fulfilled without MPPT, the TEG system was highly affected by load changes and the highest power was 
achieved with the load value about $7 \Omega$, which was the impedance matching value as expected. The experimental and the simulation results having the PSO MPPT algorithm embedded in the boost converter are compared in the given below. The obtained power in the application with MPPT was not affected very much by the load variations and the power was close to the impedance matching.

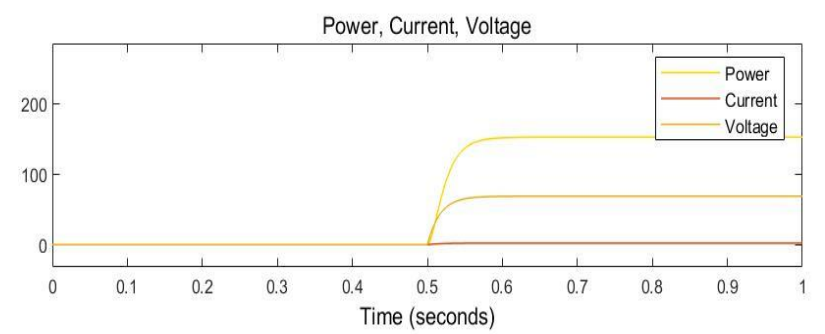

Fig. 9. Waveforms for TEG system modeling without a PSO MPPT boost converter

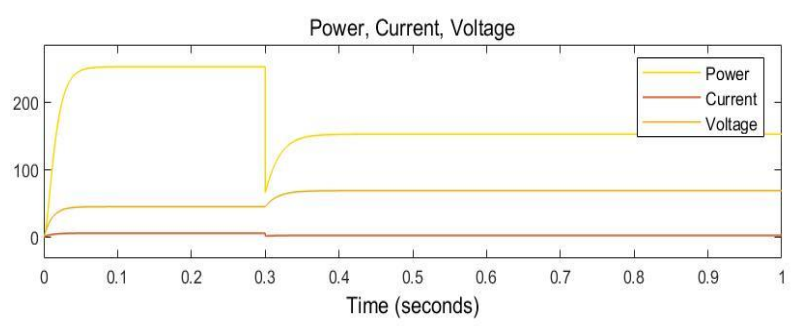

Fig. 10. Waveforms for TEG system modeling with a PSO MPPT boost converter

\section{Conclusion}

In this study, the modeling of TEGs via MATLAB/Simulink was presented depending on the Seebeck coefficient and temperature values provided by TEG manufacturers. The modeling was designed in such a way that the quantities of TEGs connected in series and parallel could be entered. In addition, both the direct load connection and the load connection with the PSO MPPT boost converter models were carried out to emphasize the importance of impedance matching between the load and TEG internal resistance. Although the modeling without PSO MPPT obtained the highest power on the impedance matching, the highest value was far from this power value for other load values. Furthermore, $98.54 \%$ of the TEG power was transferred to the output by means of the proposed boost converter with PSO MPPT. The proposed TEG and PSO MPPT boost converter models were confirmed by means of an experimental liquid-liquid TEG system setup. Consequently, both the TEG and the PSO MPPT boost converter models were presented for TEG users.

\section{References}

[1] B. H. Khan, "Non-conventional energy resources," McGraw Hill publication, 2nd Edition, 2012.

[2] https://www.nrel.gov/pv/perovskite-organic-photovoltaics.html

[3] R. Agrawal, and S. Jain, "Multilevel inverter for interfacing renewable energy sources with low/medium- and high-voltage grids," IET Renewable Power Generation, vol. 11, no. 14, 2017.

[4] "Statistics Related to Climate Change - India 2015", Social Statistics Division, Ministry of Statistics \& Programme Implementation.

[5] "Transitions in Indian Electricity Sector 2017-2030", The Energy and Resources Institute (TERI), 2017.

[6] http://indianpowersector.com/tag/per-capita-energy-consumption/.

[7] "All India-Region Wise Power Generation Overview 30-Jun-2018," Central Electricity Authority, India, Sub-Report-1, pp. 1.

[8] "Draft National Electricity Plan", vol. 1, Dec. 2016.

[9] A. Gupta, P. Kumar, R. K. Pachauri, and Y. K. Chauhan, "Performance analysis of neural network and fuzzy logic based MPPT techniques for solar PV systems," in Power India International Conference (PIICON), 2014, 6th IEEE, pp. 1-6.

[10] J. Lagorse, D. Paire, and A. Miraoui, "Sizing optimization of a standalone street lighting system powered by a hybrid system using fuel cell, PV and battery", in Renewable Energy, vol. 34, no. 3, pp. 683-691, 2009.

[11] W. X. Shen, "Optimally sizing of solar array and battery in a standalone photovoltaic system in Malaysia", in Renewable Energy, vol. 34, no. 1, pp. 348-352, 2009.

[12] P. Chitra, and R. Seyezhai, "Basic design and review of two phase and three phase interleaved boost converter for renewable energy sources," International journal of applied science.

[13] D. Hart, "Power Electronics," Tata McGraw Hill Edition 2011, New York.

[14] S. S. Mohammed, D. Devaraj, and T. I. Ahamed, "Modeling, simulation and analysis of photovoltaic modules under partially shaded conditions," in Indian Journal of Science and Technology, vol. 9, no. 16, pp. 1-8, 2016. 